James Meredith • Hoi Dick Ng • John H.S. Lee

\title{
Detonation diffraction from an annular channel
}

Submitted: 28 August 2009; Revised: 15 March, 2010

\begin{abstract}
In this study, gaseous detonation diffraction from an annular channel was investigated with a streak camera and the critical pressure for transmission of the detonation wave was obtained. The annular channel was used to approximate an infinite slot resulting in cylindrically expanding detonation waves. Two mixtures, stoichiometric acetylene-oxygen and stoichiometric acetyleneoxygen with $70 \%$ Ar dilution, were tested in a 4.3 and $14.3 \mathrm{~mm}$ channel width $(W)$. The undiluted and diluted mixtures were found to have values of the critical channel width over the cell size around 3 and 12 respectively. Comparing these results to values of the critical diameter $\left(d_{c}\right)$, in which a spherical detonation occurs, a value of critical $d_{c} / W_{c}$ near 2 is observed for the highly diluted mixture. This value corresponds to the geometrical factor of the curvature term between a spherical and cylindrical diverging wave. Hence, the result is in support of Lee's proposed mechanism [Dynamics of Exothermicity, pp. 321 (1996)] for failure due to diffraction based on curvature in stable mixtures such as those highly argon diluted with very regular detonation cellular patterns.
\end{abstract}

Keywords Gas phase detonation · diffraction · annular channel $\cdot$ critical channel width

PACS 47.40. Rs

\section{Introduction}

When a detonation wave propagating down a rigid round tube exits the tube into open space filled with the same reactive mixture, the detonation will fail if the tube has

J. Meredith · J.H.S. Lee

Mechanical Engineering, McGill University

Montreal, Quebec, H3A 2K6, Canada

H.D. Ng

Mechanical and Industrial Engineering, Concordia University

Montreal, Quebec, H3G 1M8, Canada

Tel.: (514) 848-2424 x 3177

Fax: (514) 848-3175

E-mail: hoing@encs.concordia.ca a diameter less than some critical value, referred to as the critical diameter, $d_{c}$. Should $d>d_{c}$, the detonation will re-initiate and continue to propagate once it exits its previous confinement [1]. Similar to initiation energy, this dynamic parameter can be determined much more precisely than the detonation cell size. It can serve as an alternative length scale that could provide an assessment of the relative detonation sensitivity of combustible mixtures. The phenomenon itself is also of great fundamental significance containing all the essential mechanisms for failure and initiation.

Many experiments had been carried out in the past for the critical tube diameter problem [2-4]. Critical tube diameter of common fuel-air mixture in general can be determined roughly from the empirical correlation $d_{c}=$ $13 \lambda$ where $d_{c}$ is the critical tube diameter and $\lambda$ is the characteristic detonation cell size [2]. Although this empirical correlation appears to be quite adequate for most gaseous hydrocarbon explosives, it is shown to be invalid for mixtures with regular cell patterns such as mixtures with high argon dilution [4-7]. The correlations ranged from $d_{c}=25-30 \lambda$. This breakdown clearly indicated the distinct differences in the critical diameter phenomenon for the undiluted and highly diluted mixtures.

Studies have indicated that the detonation stability or cell regularity may play an important role in the detonation undergoing a sudden expansion into open space. Lee proposed two mechanisms: one local and one global for the diffracted wave to explain the failure and the breakdown of the $13 \lambda$ rule [4]. The $13 \lambda$ rule is generally applicable for mixtures with high degree of instability (i.e. mixture reactivity highly sensitive to temperature) and the failure stems from the inability to form local explosion centers in the failure wave when it has penetrated to the charge axis. The breakdown of the $13 \lambda$ correlation is ascribed to the regularity of the cell pattern, typical for highly argon diluted mixtures. The failure in this case occurs due to the global mechanism when the curvature of the attenuated detonation exceeds some critical value required for self-sustained propagation. 
Detonation diffraction from a rectangular channel into free space, in which the aspect ratio $L / W \rightarrow \infty$, results in a cylindrical expansion of the detonation wave. In this case, only the width of the slot has an effect on the detonation and a critical width, $W_{c}$, can be defined in the same way as the critical diameter $d_{c}$. In this study, critical widths for the successful transformation of a planar to a cylindrical detonation are measured in different stable and unstable mixtures. Again, detonations in mixtures that are highly diluted with argon are considered as 'stable' in that the cellular detonation patterns are observed to be highly regular. By stable detonations we also referred to the propagation mechanism being that of shock induced ignition as described by the classical ZND model. Unstable detonations in undiluted hydrocarbon mixtures referred to those that instabilities and turbulence play the dominant role in the reaction zone and on the propagation mechanism; the cellular patterns are generally very irregular. A thin annular channel is used rather than a thin rectangular slot in order to avoid edge effects from the side wall. The width of the channel is small compared to the radius of the annulus such that radial curvature effects are negligible and a twodimensional geometry can be realized. The objective of this work is to establish and examine the critical channel width correlation with cell size for both unstable and stable mixtures. By determining the values of $W_{c} / \lambda$ and comparing the ratio $d_{c} / W_{c}$, it is possible to gain further understanding of the failure mechanism in the combustibles tested as well as the effects of geometry on detonation propagation and diffraction. According to Lee [4], if the mechanism of failure for stable mixtures is based on curvature, this should result in a scaling relationship between $d_{c}$ and $W_{c}$. Simple geometric considerations of the governing equations predict that the curvature of a cylindrical surface is half that of a spherical one, i.e., a detonation in a channel has curvature in only one plane while a detonation in a circular tube has curvature in two orthogonal planes, therefore the theory expects the critical channel width should be half the critical diameter $W_{c} \approx d_{c} / 2$, a geometrical factor of two.

\section{Experimental details}

A $3.0 \mathrm{~m}$ long steel detonation tube with an inner diameter of $65 \mathrm{~mm}$ was used and the annular geometry was affixed to the end. A Chapman-Jouguet (CJ) detonation was first generated in the round steel tube which propagates for $2.64 \mathrm{~m}$ before it entered a $360 \mathrm{~mm}$ long annular channel inserted in the detonation tube. The annulus hubs made of brass have varying diameters to create different annular channel widths. Two different channel widths were considered in this study, i.e. $W=4.3$ and $14.3 \mathrm{~mm}$. These brass inserts in the annular channel section are held in position by two sets of three equally spaced fins around the circumference. These fins are 50

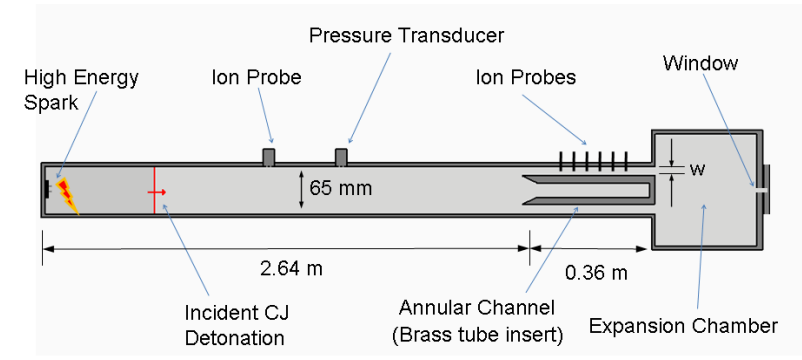

Fig. 1 A schematic of the experimental setup

$\mathrm{mm}$ long and $1 \mathrm{~mm}$ thick, which provide a rigid support of the brass inserts. Their leading and trailing edges have all been sharpened and chamfered to reduce their interference with the flow or to prevent creating any wave process from affecting the propagation of the detonation wave inside the channel. The fins also end $65 \mathrm{~mm}$ away from the end of the channel. This distance is several times the cell size and as such, the effect of the fins on the detonation by the time it arrives at the plane of expansion is assumed to be negligible. For each setup, a Vernier Caliper (digital) was used to measure the channel width at different location along the circumference to ensure that the channel width is within the accuracy of the Vernier Caliper $( \pm 0.01-0.02 \mathrm{~mm})$. The end of the annular section was then connected to the expansion chamber. The chamber is $30 \mathrm{~cm}$ long and $15.3 \mathrm{~cm}$ in diameter. It has a window in the end flange to allow streak photographs to be taken looking head-on the propagation of the diffracted detonation waves. A slit height of 1.5 $\mathrm{mm}$ was used. A sketch of the experimental apparatus is shown in figure 1.

Stoichiometric mixtures of acetylene-oxygen and stoichiometric mixtures of acetylene-oxygen with $70 \%$ argon dilution were tested. The mixtures were prepared beforehand in a separate vessel by the method of partial pressures. The gases were allowed to mix in the vessel for at least $24 \mathrm{hrs}$ in order to ensure homogeneity. For any given experiment, the tube and chamber were initially evacuated to approximately $100 \mathrm{~Pa}$. The entire apparatus was then filled from both ends to the desired initial pressure. The sensitivity of the mixtures was varied by the initial pressure. Direct initiation of a detonation was achieved by means of a high energy spark. In some cases, a long detonation driver section filled with stoichiometric acetylene-oxygen at $40 \mathrm{kPa}$ was used to directly initiate the test mixture. The driver mixture was separated from the test mixture by a thin Mylar diaphragm.

Ionization probes were used in both the detonation tube and annular channel sections to ensure a fully selfsustained detonation is obtained in the smooth round tube and annulus sections. A rotating drum streak camera with a constant film speed of about $80 \mathrm{~m} / \mathrm{s}$ was used to obtain the velocity of the diffracted wave propagating in the large chamber. 

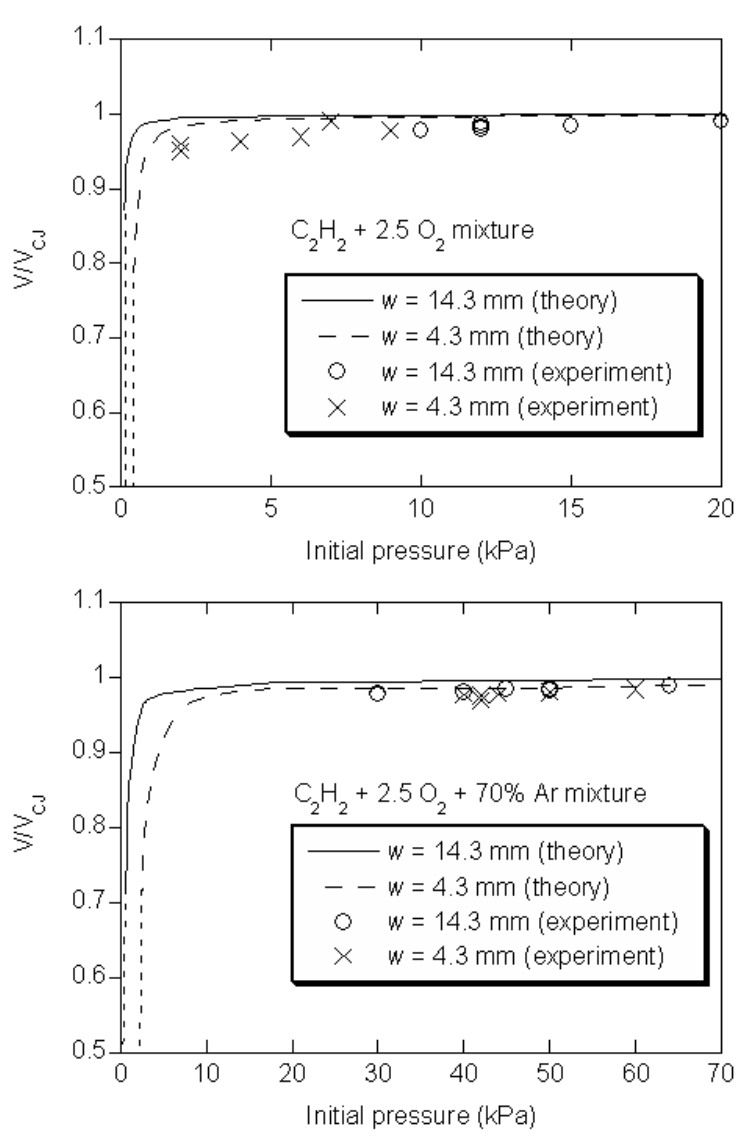

Fig. 2 Velocity deficits with comparison of theoretical model

\section{Results and discussion}

It is important to first confirm if a detonation propagates in the annular channel prior to the diffraction process. Figure 2 shows the normalized velocity $\left(V / V_{C J}\right)$ of the detonation wave propagating inside the annular channel as a function of initial pressure. These data were obtained from two ion probes inserted into the annular section to measure the speed of the detonation waves in the channel. Theoretical prediction using Fay's quasisteady model of boundary layer effect, which is shown to provide a reasonable estimate for the velocity deficit and limits for diluted/undiluted acetylene-oxygen mixtures [8], is also plotted in figure 2. From both experimental data and theoretical results, one can see that the velocity deficit for the initial pressures considered is very negligible and hence, in all experimental conditions used in this study, a detonation is still propagating inside the channel at essentially CJ speed before the diffraction process.

As discussed in previous section, streak photographs were taken looking head-on to the propagation of the diffracted detonation waves through the slit of the window in the end flange of the expansion chamber (see figure 3 ). The streak camera records an image on a sin-

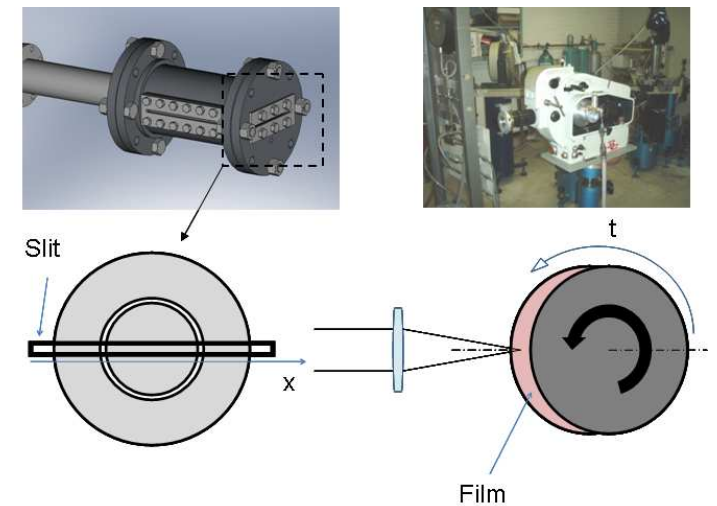

Fig. 3 The streak photography setup

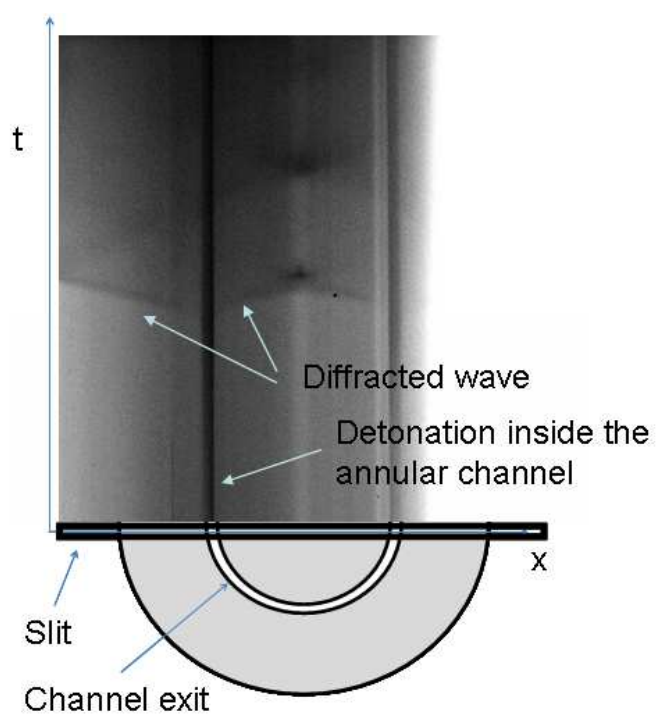

Fig. 4 The streak photography with different features labeled

gle spatial axis. The one-dimensional image is 'streaked' across the film loaded onto the rotating drum in the camera, thus recording a time history of the image on the spatial axis. The aforementioned effect can be illustrated in figure 4 with some key labels to help interpreting the streak photograph.

Typical streak photograph of a self-sustained detonation that was successfully re-established and of a failed diffracted wave that decayed abruptly upon exiting the channel are illustrated in figures 5 and 6 for the undiluted and diluted mixture cases, respectively. To classify a detonation as showing either a failure or a successful transition, the streak image was analyzed using the following criteria.

In figure 5 , the diffracted waves under consideration are shown by the arrows. The dark spot occurring at the wave intersection is where the cylindrically expanding waves have intersected at the axis of the annulus. Once this intersection occurs, more complex interactions are not considered in this investigation. In the interest of 


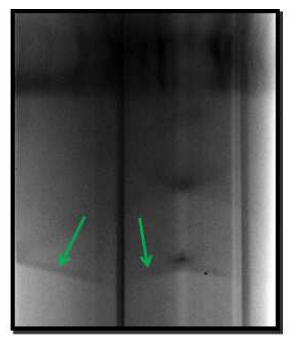

Successful Transition

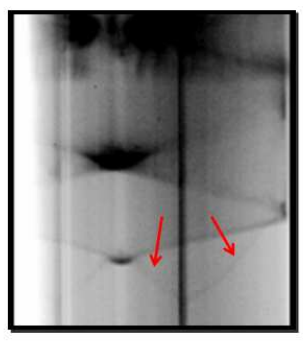

Failure
Fig. 5 Typical streak photographs for successful transition and failure in undiluted stoichiometric $\mathrm{C}_{2} \mathrm{H}_{2}-\mathrm{O}_{2}$ mixtures at $p_{o}=15 \mathrm{kPa}$ and $p_{o}=10 \mathrm{kPa}$, respectively.

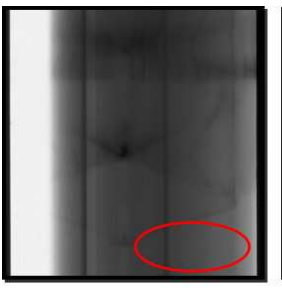

Failure $(w=4.3 \mathrm{~mm})$

Expanding waves not visible

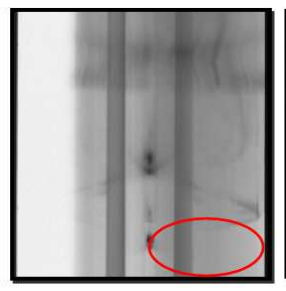

Failure $(w=14.3 \mathrm{~mm})$
not visible

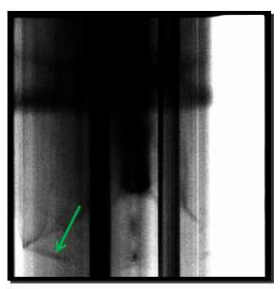

Successful Transition $(w=14.3 \mathrm{~mm})$
Fig. 6 Typical streak photographs for successful transition and failure in stoichiometric $\mathrm{C}_{2} \mathrm{H}_{2}-\mathrm{O}_{2}-70 \% \mathrm{Ar}$ diluted mixtures at $p_{o}=40 \mathrm{kPa}, 40 \mathrm{kPa}$ and $64 \mathrm{kPa}$, respectively .

examining only the cylindrical expansion, any markings on the film above this point are not considered.

For undiluted mixtures shown in figure 5 , the diffracted wave existing from the annular channels are visible in the streak photograph and the criterion to define failure and successful transition is obtained by measuring the velocity of the diffracted wave. The successfully transitioned waves, shown by the green arrows, are much more luminescent and have a much steeper angle, thus are moving faster. The velocity of these waves was found to be on the order of $V_{C J}$. In the case of failure, the waves, shown by the red arrows, are much lighter and much slower, having speeds less than $1000 \mathrm{~m} / \mathrm{s}$ and typically around 500 $\mathrm{m} / \mathrm{s}$. Typical velocity results measured from the streak photographs for a successful transition and failure are shown in figure 7 . It is important to point out that variation in the velocities computed by picking points on the streak film is quite large. This is partially due to the fact that there is an element of subjectivity in picking points manually along the streaks in the films. This method is, however, sufficient to characterize either a 'Go' or 'NoGo'.

In the highly argon diluted mixture illustrated in figure 6 , the expanding waves were not visible in the case of failure; the areas circled in red above have no visible markings where the waves should be seen. A wave that has successfully transitioned from planar to cylindrical is again shown by the green arrow above.

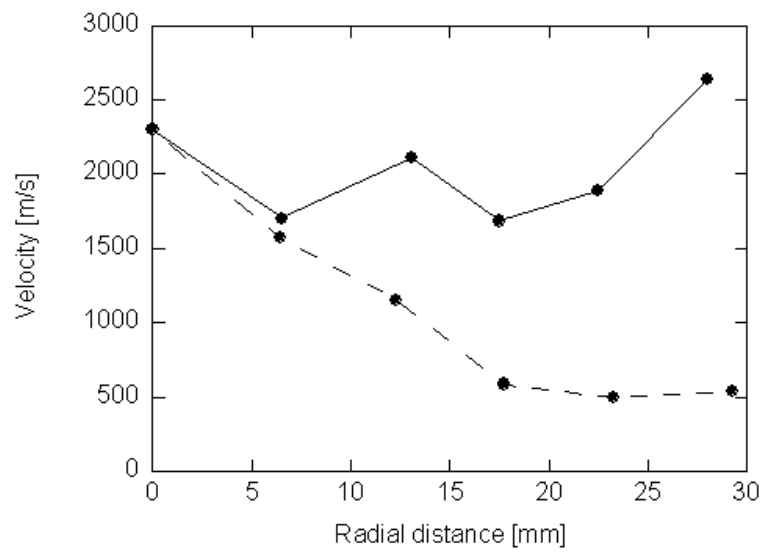

Fig. 7 Velocities of a successfully transitioned wave (solid line) and that of a failing wave (dashed line) in $\mathrm{C}_{2} \mathrm{H}_{2}+2.5$ $\mathrm{O}_{2}$ as it expands from the $4 \mathrm{~mm}$ annular channel.

The results for successful and unsuccessful transition from a planar detonation propagating in the annular channel to a cylindrical detonation in the large chamber are given in figures $8 \mathrm{a}, \mathrm{b}$ as a function of initial pressures for different mixtures with and without Ar dilution, respectively. The critical pressure for each annulus and mixture lies between an adjacent failure $(\mathrm{X})$ and successful transmission $(\mathrm{O})$. These plots show that no critical pressure was found for $\mathrm{C}_{2} \mathrm{H}_{2}+2.5 \mathrm{O}_{2}+70 \% \mathrm{Ar}$ in the $4 \mathrm{~mm}$ channel as it lies above the maximum initial pressure accessible to the equipment used in this study. Values of the critical presure determined from the figures 8 are summarized in table 1.

Values of cell size $\lambda$ with initial pressure for the two acetylene-oxygen mixture conditions are taken from [9][10]. The resulting power-law best fit correlation between cell size and initial pressure are given as:

$\lambda[\mathrm{mm}]=a \cdot\left(p_{o}[\mathrm{kPa}]\right)^{b}\left\{\begin{array}{c}a=23.8 b=-1.03 \text { for } 0 \% \mathrm{Ar} \\ a=113.8 b=-1.20 \text { for } 70 \% \mathrm{Ar}\end{array}\right.$

Using the above correlation, the corresponding cell sizes $\lambda$ for the critical pressures can be obtained and are also summarized in table 1. Based on these results, some important observations can be made. For the results of unstable mixtures (undiluted stoichiometric $\mathrm{C}_{2} \mathrm{H}_{2}-\mathrm{O}_{2}$ mixtures), the critical channel width over detonation cell size for the two channels used are found to be about 2.74 3.80. This result agrees well with that of Liu et al. [11] where they observed that as the expansion of the detonation approaches cylindrical form, namely for a rectangular channel with $L / W \rightarrow \infty, W_{c} / \lambda \approx 3$ for unstable mixtures. This result was also confirmed up to $L / W \rightarrow 40$ by Benedick et al. [12]. For highly argon diluted mixture with regular cellular pattern, the non-dimensional critical width $W_{c} / \lambda$ is computed to be about 12 .

Finally, Table 2 summarizes all different important ratios, namely, $d_{c} / \lambda$ and $W_{c} / \lambda$ obtained from previous 


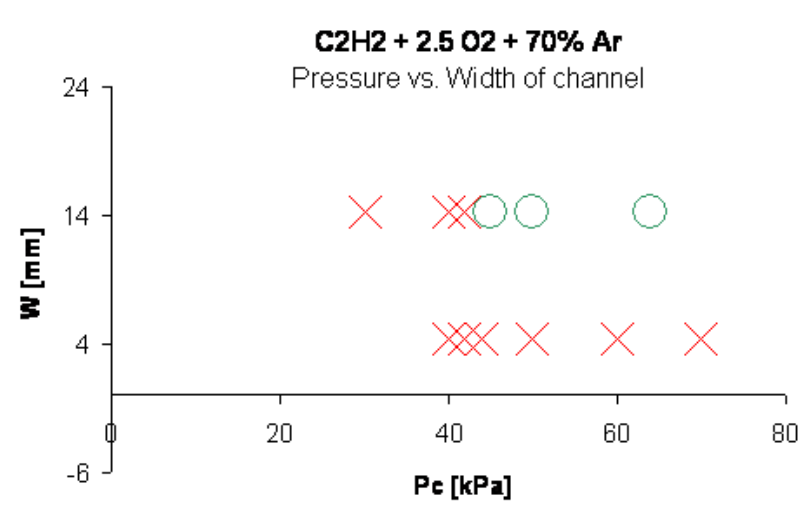

$\mathrm{C} 2 \mathrm{H} 2+2.5 \mathrm{O} 2$

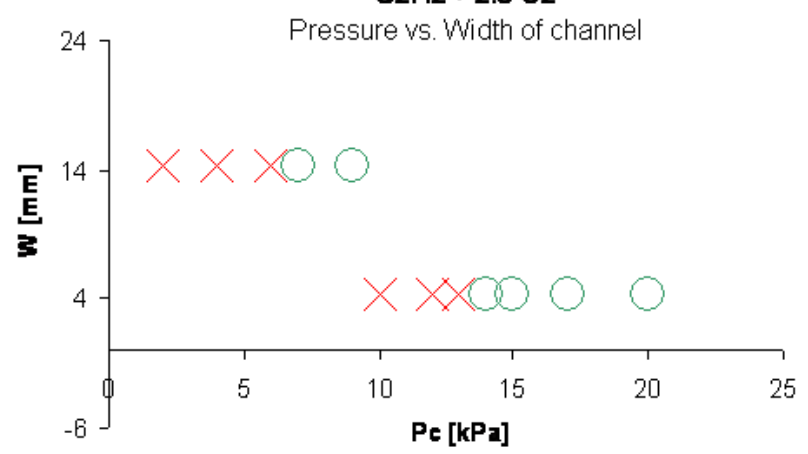

Fig. 8 Determination of the critical initial pressure from the Go/No-Go plots. X denotes failure and O denotes successful transition.

\begin{tabular}{cccc}
\hline Mixtures & $p_{c}$ & $\lambda$ & $W_{c} / \lambda$ \\
\hline $\mathrm{C}_{2} \mathrm{H}_{2}+2.5 \mathrm{O}_{2}$ & & & \\
$W=14.3 \mathrm{~mm}$ & $6 \mathrm{kPa}$ & $3.76 \mathrm{~mm}$ & 3.80 \\
$W=4.3 \mathrm{~mm}$ & $14 \mathrm{kPa}$ & $1.57 \mathrm{~mm}$ & 2.74 \\
$\mathrm{C}_{2} \mathrm{H}_{2}+2.5 \mathrm{O}_{2}+70 \% \mathrm{Ar}$ & & & \\
$W=14.3 \mathrm{~mm}$ & $45 \mathrm{kPa}$ & $1.18 \mathrm{~mm}$ & 12.1 \\
& & &
\end{tabular}

Table 1 Summary of the present experimental results

literature and the present work, and compares the critical tube diameter and the critical channel width required for a gaseous detonation to emerge and continue to propagate in an unconfined environment. As discussed earlier, Lee's proposed mechanism for failure due to excessive curvature of the entire detonation front suggest that there should be a relationship given by the geometrical factor of 2 between $d_{c}$ and $W_{c}$ for stable mixture such as $\mathrm{C}_{2} \mathrm{H}_{2}+2.5 \mathrm{O}_{2}+70 \% \mathrm{Ar}$. The present result shown in table 2 for the highly argon diluted mixture supports this proposed failure mechanism based on curvature given that a ratio of critical diameter to critical width near 2 arises for the stable mixture as predicted by front curvature theory. In the undiluted case, this factor of two does not arise, suggesting that other mechanisms not based

\begin{tabular}{lccc}
\hline & $d_{c} / \lambda$ & $W_{c} / \lambda$ & $d_{c} / W_{c}$ \\
\hline High Argon Dilution & $25-30^{*}$ & 12.1 & $2.07-2.48$ \\
Undiluted & 13 & 3.80 & 3.42 \\
& 13 & 2.74 & 4.74
\end{tabular}

Table 2 Comparison of the critical tube diameter [4-7] to the critical channel width obtained in this study.

on curvature are playing a role. The ratio $d_{c} / W_{c}$ for the unstable mixture obtained in this study is between 3.424.74 and this agrees with the study by Benedick et al. [12] where the scaling from $d_{c}$ to $W_{c}$ was found to be approximately $4: 1$.

To look at how the uncertainty in different measurement affects the results shown in tables 1 and 2, a firstorder error analysis is performed [13]. For the channel width measurement, a conservative estimate of its uncertainty is given to be $\pm 0.1 \mathrm{~mm}$. As discussed previously, the Vernier Caliper (digital) has an accuracy of $\pm 0.01-0.02 \mathrm{~mm}$. The pressure measurement should have a degree of confidence $\pm 1 \mathrm{kPa}$. It should be mentioned that one assumes the cell size correlation provides reasonable estimate and did not consider in the present analysis the statistical error in the formula obtained from [9][10]. This requires a more detailed analysis of different cell size measurement from various sources and this is beyond the scope of this simple error estimation. With these uncertainty values, it is obtained that for the unstable mixture (undiluted stoichiometric acetylene-oxygen mixture) $W_{c} / \lambda=3.27 \pm 0.48$ and for stable mixture (Argon diluted stoichiometric acetylene-oxygen mixture) $W_{c} / \lambda=12.1 \pm 0.40$. Further calculation gives $d_{c} / W_{c}$ on average a value of $4.08 \pm 0.54$ for unstable mixture, while for stable mixture with high argon dilution, this ratio $d_{c} / W_{c}$ is approximately $2.28 \pm 0.075$ The latter, within the uncertainties in measurement, is still in good agreement with the geometrical factor of 2 .

\section{Conclusion}

In this study, simple experiment to determine the critical width in acetylene-oxygen mixtures with and without argon dilution are performed by observing, by streak photography, detonations expanding from an annular channel. The annulus was used to approximate an infinite channel and achieve a cylindrically expanding detonation wave. It is found that for unstable mixture (without dilution), the correlation of critical channel width with detonation cell size is about $W_{c} / \lambda \approx 3$. As for stable mixtures (e.g. mixtures with high Ar dilution), the correlation appears to be about $W_{c} / \lambda \approx 12$.

The present study on the critical channel width provides some important confirmation on the failure mechanisms proposed by Lee on the phenomena of critical 
tube diameter problem. By comparing the critical channel width for diffracted detonations to previous data for the critical diameter, a factor near 2 arose for the mixture highly diluted with argon (where it has been suggested that the cellular instabilities play minor roles in the detonation propagation mechanism). This result appears to support a curvature based global mechanism for diffraction failure in these stable mixtures where the scaling from $d_{c}$ to $W_{c}$ is $2: 1$, corresponding to the geometrical factor of the curvature term between a spherical and cylindrical diverging wave. At last, it is worth to mention that such failure mechanism by global curvature and geometric scaling can also be observed from the experiments of detonation wave failure in porous wall tubes [14] and propagation limits of high condensed phase explosives with very fine-scale heterogeneities [15].

Acknowledgements This work was supported by the Natural Sciences and Engineering Research Council of Canada (NSERC).

\section{References}

1. Lee, J.H.S.: Dynamic parameters of gaseous detonations. Ann. Rev. Fluid Mech. 16, 331-336 (1984).

2. Mitrofanov, V.V., Soloukhin, R.I.: The diffraction of multifront detonation waves. Soviet Physics-Doklady 9 (12), 1055 (1965).

3. Knystautas,R., Lee, J.H., Guirao, C.: The critical tube diameter for detonation failure in hydrocarbon-air mixtures. Comb. Flame 48, 63-83 (1982).

4. Lee, J.H.S.: On the critical tube diameter. In: J. Bowen, Editor, Dynamics of Exothermicity, Gordon and Breach, Amsterdam, Netherlands, pp. 321 (1996).

5. Moen, I., Sulmistras, A., Thomas, G.O., Bjerketvedt, D., Thibault, P.A.: Influence of cellular regularity on the behavior of gaseous detonations. Prog. Astronautics and Aeronautics 106, 220 (1986).

6. Shepherd, J.E., Moen, I., Murray, S., Thibault, P.I. Analyses of the cellular structure of detonations. Proc. Combust. Inst. 21, 1649 (1986).

7. Desbordes, D. et al.: Failure of classical dynamic parameters relationships in highly regular cellular detonation systems. Prog. in Astro. Aero. 153, 347 (1993).

8. Chao, J., Ng, H.D., Lee, J.H.S.: Detonation limits in thin annular channels. Proc. Combust. Inst. 32, 2349-2354 (2009).

9. Radulescu, M.I.: The Propagation and Failure Mechanism of Gaseous Detonations: Experiments in Porous-Walled Tubes. Ph.D. thesis, McGill University, Canada (2003).

10. Kaneshige, M., Shepherd, J.E.: Detonation database. GALCIT Technical Report FM97 (1997). Web page at http://www.galcit.caltech.edu/detn_db/html/db.html.

11. Liu, Y.K., Lee, J.H., Knystautas, R.: Effect of geometry on the transmission of detonation through an orifice. Combust. flame, 56, 215 (1984).

12. Benedick, W., Knystautas, R., Lee, J.H.: Large scale experiments on the transmission of fuel-air detonation from two-dimensional channels. Prog. Astro. Aero. 94, 546-555 (1983).

13. Atkinson, K.E., Han, W.: Elementary Numerical Analysis. 3rd ed., Wiley (2003).

14. Radulescu, M.I., Lee, J.H.S.: The failure mechanism of gaseous detonations: Experiments in porous wall tubes. Combust. flame, 131, 29-46 (2002).
15. Petel, O.E., Mack, D., Higgins, A.J., Turcotte, R., Chan, S.K.: Minimum propagation diameter and thickness of high explosives. J. Loss Prev. Proc. Ind. 20, 578-583 (2007). 